\title{
THE SNAKE RING
}

\section{A Feasibility Study of a Small Predamping Ring for the Positron Source of SLC*}

\author{
KLAUS WILlE \\ Stanford Linear Accelerator Center \\ Stanford University, Stanford, California 94305
}

\begin{abstract}
- In order to reduce the large transverse emittances of the positron beam, as well as the large energy spread, a radiation damping time of the order of $10 \mathrm{msec}$ is required. By use of a specially designed "SNAKE"-wiggler with a field of 2 Tesla one can obtain this short damping time at a beam energy of $100 \mathrm{MeV}$. Because of the strong bending field of the wiggler, the optics of the small model predamping ring presented here, in particular the chromaticity, is mainly determined by the edge focusing at the entrance and exit of each wiggler section. In order to get a uniform damping partition the dispersion function has to have a particular shape which defines the most important optical constraint.
\end{abstract}

Submitted to Nuclear Instruments and Methods

* Work supported by the Department of Energy, contract DE-AC03-76SF00515 


\section{Introduction}

In order to produce the desired number of positrons in the SLAC Linear Collider $[1,2]$ a single bunch of electrons is extracted from the linac at the $2 / 3$ point and brought onto the tungsten target. The positrons generated are collected by means of a flux concentrator and accelerated up to $200 \mathrm{MeV}$ in a booster linac section. They are then transported back to the beginning of the lin ac, accelerated to $1.2 \mathrm{GeV}$ and then sent to the damping ring [3]. The overall positron-production system is required to have a yield of at least one positron for each incident electron in order to obtain the desired collider luminosity.

This yield is mainly restricted by the large transverse emittance and the large momentum spread of the positron beam emerging from the target. Calculations predict that the beam emittance is $5 \mathrm{MeV}-\mathrm{mm}$ in both planes and the momentum is preferentially in the $2-20 \mathrm{MeV}$ range. Given these large values it is obviously a problem to capture and to transport the desired number of particles through the entire system.

Therefore the idea came up to insert a small low-energy storage ring with high radiation damping very close to the $e^{+}$source. With such a device both the transverse emittance and the momentum spread could be reduced.

The main parameter of this small predamping ring is the damping constant of the transverse and longitudinal particle oscillation, given by the relation [4]

$$
a \sim \frac{U_{0}}{T_{0} \cdot E_{0}}
$$

$U_{0}$ is the energy loss per turn due to synchrotron radiation, $T_{0}$ the revolution time and $E_{0}$ the beam energy. Assuming a constant bending field $B$, and consequently a constant bending radius $\rho$, the energy loss per turn can be expressed as

$$
U_{0} \sim E_{0}^{4} \oint \frac{d s}{\rho^{2}}=E_{0}^{4} \int_{b e n d s} \frac{d s}{\rho^{2}}=E_{0}^{4} \cdot \frac{L_{b}}{\rho^{2}}
$$

where $L_{b}$ is the orbit length inside the bending magnets. Since $1 / \rho \sim B / E_{0}$ and $T_{0}=L / c(L=$ length of the orbit, $c=$ velocity of light $)$ the damping constant 
finally becomes

$$
a \sim E_{0}^{2} \cdot B^{2} \cdot \frac{L_{b}}{L}
$$

To achieve a strong damping at a given energy $E_{0}$, the bending field has to be as strong as possible and the ratio $L_{b} / L$ should be close to one. This means that most of the orbit lies inside a bending magnet.

On the other hand a uniform damping partition is required which is mainly determined by the integral

$$
D=\frac{1}{\rho L_{b}} \int_{b e n d s} \eta(s) d s
$$

where $\eta(s)$ is the dispersion function. The integral is taken over all bending magnets. A proper damping partition requires the condition $D \ll 1$ which can only be obtained by inserting special focusing elements and drift spaces. This increases $L$ without changing $L_{b}$ and leads to a reduction in the damping rate.

The way out is to use a wiggler-type magnet which bends the particle orbit alternately in opposite directions causing a total bending angle $\psi_{b}$ which is much larger than $2 \pi$. Defining the ratio

$$
n=\frac{\psi_{b}}{2 \pi} \quad(n \gg 1)
$$

the energy loss per turn becomes instead of eq. (2)

$$
U_{0} \sim n \cdot E_{0}^{4} \oint \frac{d s}{\rho^{2}}
$$

The resulting damping constant $a$ is also $n$ times larger.

The feasibility of a small predamping ring has been investigated using the design principles sketched above. The results are presented in this paper. The beam energy is chosen to be $E_{0}=100 \mathrm{MeV}$ which gives a reasonable damping time using only conventional techniques. 


\section{The Wiggler Magnet}

A standard wiggler magnet is a periodic structure of short bending magnets with alternating field polarities (fig. 1). If $B_{\text {pole }}$ is the flux density at the wiggler poles, the maximum field on the beam axis is [5]

$$
B_{0}=\frac{B_{\text {pole }}}{\cosh \frac{2 \pi D}{\lambda}}
$$

where $D$ is the distance between pole and beam axis and $\lambda$ is the period length. In order to get strong damping, $B_{0}$ should be as high as possible and is supposed not to be significantly smaller than $B_{\text {pole }}$. Therefore for a given pole distance $2 \cdot D$, which is determined by the beam dimension, a minimum period length $\lambda$ is required. For instance, if $B_{0} \geq 0.9 B_{\text {pole }}$ and $D=2 \mathrm{~cm}$, one can derive from (7) a period length which is supposed to be at least $\lambda \geq 27 \mathrm{~cm}$. With this value, the maximum possible wiggler field using conventional iron magnets is approximately $B_{0} \simeq 1.8 \mathrm{~T}$.

- On the other hand, the bending radius of a $100 \mathrm{MeV}$ electron beam passing through a magnet field $B_{0}=1.8 \mathrm{~T}$ is only $\rho=18.5 \mathrm{~cm}$ which is of the order of the period length. Consequently the beam is bent transversely by a large amount and has a significant angle $\Theta$ with respect to the pole faces (fig. 1). The resulting edge focusing which is proportional to the ratio $\tan \theta / \rho$ is extremely high and dominates the beam optics. Because of these reasons it was not possible to find a proper solution using a standard wiggler. Therefore, a special wiggler magnet has been designed which basically consists of two long C-magnets of opposite polarities mounted facing each other (fig. 2). A beam entering the magnet A perpendicular to the pole face will follow a half-circle inside the magnet and will also exit perpendicularly to the pole face because of geometrical reasons. The same happens in the magnet $\mathrm{B}$ but with opposite bending direction. This scheme provides only right angles between beam and magnet poles avoiding the edge focusing problems mentioned above. Because of the snake-like orbit, this magnet is called "SNAKE wiggler." 


\section{Magnet Structure and Optics of the Small Predamping Ring}

The magnet structure of the model predamping ring suggested in this paper essentially consists of two SNAKE wigglers providing a total bending angle of $10 \cdot \pi$. As shown in fig. 3 , the ring has a fourfold symmetry and two longer drift-spaces necessary because of optical reasons and also used for injection elements and the rf-cavity. The two wigglers are combined in one integrated magnet sketched in the cross section of fig. 4 .

In the horizontal plane the weak focusing in the bending magnets which is proportional to $1 / \rho^{2}$ is a dominant contribution and cannot be changed without changing the geometry of the ring. Therefore the pole face at the entrance and exit of each wiggler is rotated providing an additional variable horizontal focusing $(Q F)$. In the vertical plane two short standard quadrupoles are mounted in the middle of each drift space. Further parameters concerning mainly the geometrical structure of the ring are listed in the following table:

Table 1. Magnet and Geometrical Data of the Predamping Ring

\begin{tabular}{ll}
\hline Bending field (in the wiggler) & $B=2.0 \mathrm{~T}$ \\
Bending radius (at $E_{0}=100 \mathrm{MeV}$ ) & $\rho=0.16678 \mathrm{~m}$ \\
Length of the long driftspace & $\ell_{1}=0.3246 \mathrm{~m}$ \\
Length of the driftspaces in the SNAKE wiggler & $\ell_{2}=0.05 \mathrm{~m}$ \\
Length of the quadrupole $Q D$ & $\ell_{Q}=0.10 \mathrm{~m}$ \\
Orbit length & $L=7.13801 \mathrm{~m}$ \\
Orbit length inside the bending field & $L_{b}=5.2396 \mathrm{~m}$ \\
& $L_{b} / L=0.734$ \\
Revolution time & $T_{0}=23.81 \mathrm{nsec}$ \\
Revolution frequency & $f_{u}=42.00 \mathrm{MHz}$ \\
\hline
\end{tabular}

The predamping ring should have approximately the same damping in both transverse planes and also a short damping time in the energy phase space. Therefore, one has to look very carefully at the damping partition. The damping constants of the energy and the transverse betatron oscillations, respectively, are normally 
expressed by the following formula [4]:

$$
\begin{aligned}
& a_{E}=\frac{U_{0}}{2 T_{0} E_{0}}(2+D) \\
& a_{x}=\frac{U_{0}}{2 T_{0} E_{0}}(1-D)
\end{aligned}
$$

where $D$ has been defined by eq. (4).

In order to get proper damping in all planes, one can easily see from (8) and (9) the following constraint

$$
0 \ll 1
$$

This means because of (10) the dispersion $\eta(s)$ inside the bending magnets has to be either very small or it has to oscillate with respect to the orbit. We will see that only the second case is possible.

The transformation of the dispersion through a bending magnet may be expressed as a matrix equation:

$$
\left(\begin{array}{c}
\eta(s) \\
\eta^{\prime}(s) \\
1
\end{array}\right)=\left(\begin{array}{ccc}
\cos \frac{s}{\rho} & \rho \cdot \sin \frac{s}{\rho} & \rho\left(1-\cos \frac{s}{\rho}\right) \\
-\frac{1}{\rho} \sin \frac{s}{\rho} & \cos \frac{s}{\rho} & \sin \frac{s}{\rho} \\
0 & 0 & 1
\end{array}\right)\left(\begin{array}{c}
\eta_{0} \\
\eta_{0}^{\prime} \\
1
\end{array}\right)
$$

where $s$ describes the distance to the magnet entrance along the orbit. In particular the dispersion function becomes

$$
\eta(s)=\left(\eta_{0}-\rho\right) \cos \frac{s}{\rho}+\eta_{0}^{\prime} \cdot \rho \cdot \sin \frac{s}{\rho}+\rho
$$

With this expression the integral of the dispersion inside a bending magnet has the following simple analytical form

$$
\int_{0}^{\ell} \eta(s) d s=\rho\left(\eta_{0}-\rho\right) \sin \frac{\ell}{\rho}+\eta_{0}^{\prime} \rho^{2}\left(1-\cos \frac{\ell}{\rho}\right)+\rho \ell
$$


Let $\ell=\pi \cdot \rho$ be the length of a half-circle, i.e., the length of the orbit in a $180^{\circ}$ bending magnet, expression (13) reduces to

$$
\int_{0}^{\ell} \eta(s) d s=\rho^{2}\left(2 \eta_{0}^{\prime}+\pi\right)
$$

It is remarkable that the integral and consequently the damping partition only depends on the derivative $\eta_{0}^{\prime}$ of the dispersion at the entrance of the bending magnet. The value $\eta_{0}$ at this point has no influence. Generally, in a structure like the SNAKE wiggler with a series of $180^{\circ}$-magnets with alternating direction of the bending field, the integral of the dispersion in the $i^{\text {th }}$ magnet becomes

$$
\int_{i^{t h} \text { bend }} \eta_{i}(s) d s=(-1)^{i+1} \cdot \rho^{2}\left(2 \eta_{0}^{\prime}+\pi\right)
$$

where $\eta_{0}^{\prime}$ defines the initial value at the first magnet. With this simple expression we can finally write the integral (4) of the entire ring as

$$
D=\frac{2 \rho}{L_{b}}\left(2 \eta_{0}^{\prime}+\pi\right)=\frac{1}{5 \pi}\left(2 \eta_{0}^{\prime}+\pi\right)
$$

In this equation the orbit length inside all bending magnets of the predamping ring is $L_{b}=10 \pi \rho$ due to the total bending angle of $\psi_{0}=10 \pi$. One can easily see that $D$ vanishes if

$$
\eta_{0}^{\prime}=-\frac{\pi}{2}
$$

On the other hand if $D$ vanishes also the momentum compaction factor

$$
\alpha=\frac{1}{L} \oint \frac{\eta(s)}{\rho(s)} d s=\frac{1}{\rho \cdot L} \int_{b e n d s} \eta(s) d s
$$

vanishes. The resulting synchrotron frequency $f_{s}$ as well as the bunch length $\sigma_{s}$ becomes zero which is obviously not desirable. Therefore the bunch length is arbitrarily set to $\sigma_{s}=3 \mathrm{~mm}$ which requires a momentum compaction factor of $\alpha=$ $8.5 \times 10^{-3}$. With this additional condition the required derivative of the dispersion at the entrance of the SNAKE wiggler is because of (15) and (18)

$$
\eta_{0}^{\prime}=\frac{1}{2}\left(\frac{\alpha L}{2 \rho}-\pi\right) \approx-1.48 \mathrm{rad}
$$


The $\eta_{0}^{\prime}$ value is, however, not very critical. Even with $\eta_{0}^{\prime}=0$ expression (16) provides $D=0.2$ which is tolerable from the damping partition point of view.

A possible optics for the SNAKE damping ring satisfying this constraint has been developed and is plotted for one-half ring in fig. 5. Because the horizontal weak focusing dominates inside the bending magnets and this does not act in the vertical plane, the optics of both planes are quite different. This results in a large difference between the horizontal and vertical tunes.

The following list contains the most important parameters of the damping ring optics:

Table 2. Optics Parameters of the SNAKE Damping Ring.

\begin{tabular}{ll}
\hline Pole face rotation $(Q F)$ & $\Theta_{\text {rot }}=-22.609$ degree \\
Quadrupole strength $(Q D)$ & $k=30.029 \mathrm{~m}^{-2}$ \\
Tune & $\nu_{x}=5.80$ \\
$\nu_{z}$ & $=0.74$ \\
Momentum compacting factor & $\alpha=8.5 \times 10^{-3}$ \\
Equilibrium emittance & $\epsilon_{x}=0.382 \pi \mathrm{mm} \mathrm{mrad}$ \\
Energy loss per turn & $U_{0}=265.3 \mathrm{eV}$ \\
Damping constants & $a_{E}=112.07 \mathrm{sec}^{-1}$ \\
& $a_{x}=55.07 \mathrm{sec}^{-1}$ \\
& $a_{z}=55.71 \mathrm{sec}^{-1}$ \\
Damping times & $\tau_{E}=8.92 \mathrm{msec}$ \\
& $\tau_{x}=18.16 \mathrm{msec}$ \\
& $\tau_{z}=17.95 \mathrm{msec}$ \\
\hline
\end{tabular}

The calculated damping times are of the order of $10 \mathrm{msec}$ which is very small for a storage ring at an energy of $E=100 \mathrm{MeV}$. If $\Delta E_{0}$ and $\epsilon_{0}$ are the energy spread and the beam emittance respectively measured at injection, the corresponding values after a time $\Delta t$ are $\Delta E$ and $\epsilon$. Since the damping ring will run with a repetition rate of $f_{r e p}=180 \mathrm{~Hz}$ the time between injection and extraction is $\Delta t=5.56 \mathrm{msec}$. After this time the energy spread and the transverse emittance will be reduced by 
the factor

$$
\frac{\Delta E}{\Delta E_{0}}=\exp \left(-\frac{\Delta t}{\tau_{E}}\right)=0.54 \quad \text { and } \quad \frac{\epsilon_{x}}{\epsilon_{0}}=\exp \left(-\frac{2 \cdot \Delta t}{\tau_{x}}\right)=0.54
$$

\section{Compensation of the Chromaticity}

Since the predamping ring should be able to accept particles in an energy range of up to $\Delta p / p= \pm 0.1$ the chromaticity produced by the separated vertical focusing quadrupole $Q D$ is

$$
\Delta \xi_{x, z}= \pm \frac{1}{4 \pi} \cdot \beta_{x, z} k \ell
$$

where the positive sign belongs to the horizontal and the negative sign to the vertical plane respectively. $\beta$ is the average amplitude function in the quadrupole and $k \ell$ is the integrated quadrupole strength. Using the optical data the chromaticity of the quadrupoles becomes

$$
\left.\begin{array}{l}
\Delta \xi_{x}=+0.05 \\
\Delta \xi_{z}=-7.28
\end{array}\right\} \quad \text { quadrupole }
$$

But there is still another mechanism in the ring generating a large amount of chromaticity. As described above the dispersion has to have an angle of approximately $\pi / 2$ with respect to the orbit at each pole face of the SNAKE wiggler. Thus a particle with a non-zero energy deviation $\Delta p / p \neq 0$ will cross the pole face at a certain angle $\psi$ causing an energy dependent edge focusing proportional to $\tan \psi / \rho$. Since

$$
\tan \psi=\eta^{\prime} \frac{\Delta p}{p}
$$

the resulting chromaticity generated at one particular pole face is

$$
\Delta \xi_{x, z}= \pm \frac{1}{4 \pi} \cdot \beta_{x, z} \frac{\eta^{\prime}}{\rho}
$$

If the pole face is at the beam entrance, the negative sign in (23) describes the horizontal chromaticity whereas the positive sign gives the vertical value. At the 
beam exit the sign convention is reversed. The chromaticity due to this energy dependent edge focusing evaluated for the entire ring is $\Delta \xi_{x}=+16.77$ and $\Delta \xi_{z}=-7.95$. The total chromaticity of the predamping ring is therefore

$$
\begin{aligned}
& \xi_{x}=+16.82 \\
& \xi_{z}=-15.23
\end{aligned}
$$

These are extremely high values for such a small ring and a careful compensation is required which usually is done by means of sextupole magnets distributed around the ring. But in this compact little ring there is almost no space available for additional magnets. In addition the chromaticity generated at each pole face is too high to be compensated elsewhere. Therefore it seems reasonable to curve each pole face such that particles of different energies cross the pole face at the same right angle. Under these circumstances no chromaticity due to edge focusing will be generated at all.

--A particle with energy deviation $\Delta p / p \neq 0$ follows a dispersion trajectory which at the pole face has a displacement of

$$
x=\eta \frac{\Delta p}{p}
$$

with respect to the central orbit (fig. 6). If $f(x)$ represents the pole face curvature the derivative of this function has to be

$$
\frac{d f}{d x}=-\eta^{\prime} \frac{\Delta p}{p}
$$

in order to satisfy the constraint mentioned above. Taking the relation (24) one can eliminate the energy deviation $\Delta p / p$ and the differential equation of the curvature function becomes

$$
\frac{d f}{d x}=-\frac{\eta^{\prime}}{\eta} x
$$

A simple integration finally provides the desired pole face curvature

$$
f(x)=A x^{2}
$$


with

$$
A=-\frac{\eta^{\prime}}{2 \eta}
$$

Starting at the entrance of the SNAKE wiggler $(Q F)$ there are five pole faces per quadrant (fig. 3) with different dispersion values. The corresponding curvature factors (28) for the predamping ring are listed in the table below.

Table 3. Curvature Factors

\begin{tabular}{ccc}
\hline No. of pole face & Type & $A\left(m^{-1}\right)$ \\
\hline $1(Q F)$ & entrance & -0.496 \\
2 & exit & -1.139 \\
3 & entrance & 1.286 \\
4 & exit & -3.067 \\
5 & entrance & 4.423 \\
\hline
\end{tabular}

The sign convention for the factor $A$ is positive if the field boundary is convex outward (fig. 6).

The chromaticity of the separated quadrupole $Q D$ will also be compensated in the magnet itself by rotating the pole faces by an angle $\phi$. Thus a particle passing through the quadrupole at a distance $x$ with respect to the orbit will see a different magnetic length $\ell+\Delta \ell$ with

$$
\Delta \ell=x \cdot \tan \phi
$$

For a particle with $\Delta p / p \neq 0$ the distance is $x=\eta \cdot \Delta p / p$ and therefore the change in chromaticity due to the pole face rotation becomes

$$
\Delta \xi_{\text {rot }}=\frac{1}{4 \pi} \cdot \beta k \eta \cdot \tan \phi
$$

The total chromaticity of a quadrupole with rotated pole faces can easily be evaluated by adding expressions (21) and (30)

$$
\Delta \xi_{x, z}= \pm \frac{1}{4 \pi} \cdot \beta_{x, z} \cdot k(\ell+\eta \cdot \tan \phi)
$$


This portion disappears if $\ell+\eta \cdot \tan \phi=0$ or

$$
\phi=-\tan ^{-1}\left(\frac{\ell}{\eta}\right)
$$

For the mini damping ring the pole face rotation was calculated to be $\phi=\mathbf{- 4 . 4}$ degree. The entire scheme used to compensate the chromaticity of the ring is sketched in fig. 7 .

\section{Injection and Extraction}

The injection and extraction scheme consists of one pulsed kicker magnet and two septum magnets as shown in fig. 8. The extraction of the stored beam takes place simultaneously with the injection of the new beam. Because of the injected positron emittance of $\epsilon_{x, z}=50 \pi \mathrm{mm}$ rad and in particular because of the large energy spread of $\Delta p / p=0.1$ the beam has very large dimensions in the septum region especially in the horizontal plane. The values are

$$
\begin{aligned}
& \sigma_{x}=\sqrt{\epsilon_{x} \beta_{x}+\left(\eta_{x} \frac{\Delta p}{p}\right)^{2}}=95 \mathrm{~mm} \\
& \sigma_{z}=\sqrt{\epsilon_{z} \beta_{z}}=25 \mathrm{~mm}
\end{aligned}
$$

On the other hand the vertical beta functions are much larger than the horizontal values. Therefore, a vertical injection and extraction has been chosen as sketched in fig. 9.

In order to inject on axis the pulsed kicker magnet has to provide a vertical bending angle of $\alpha_{k}=7.7 \mathrm{mrad}$. Assuming a kicker length of $\ell=0.20 \mathrm{~m}$ the required bending field is $B_{k i c k e r}=0.0133 \mathrm{~T}$. Even though this is not a very high field, there might be some technical problems because of the short pulse length required. Since the revolution frequency is $f_{u}=42 \mathrm{MHz}$ the total length of the kicker pulse must not exceed a value of $T_{k}=48$ nsec which can only be obtained by use of very fast pulsing techniques. A design based on a ferrite loaded strip line is most likely to satisfy the field and speed constraints. The main cross section is 
drawn in fig. 10. For this design the maximum pulse current is $I_{k}=2113 \mathrm{~A}$ and the inductivity is $L \approx 70 \mathrm{nH}$.

In order to get a sufficient separation of the injected beam at the quadrupole $Q D$ the septum magnet must provide a bending angle of at least $\alpha_{\text {sept }}=0.23 \mathrm{rad}$. If the length is chosen to be $\ell=0.2 \mathrm{~m}$ to fit within the drift space the corresponding magnetic field strength is $B_{s e p t}=0.38 \mathrm{~T}$. A possible design of the vertically bending septum is shown in fig. 11. Assuming a coil with 18 turns the required current is $I_{\text {sept }}=3360 \mathrm{~A}$ and the inductivity becomes $L \approx 30 \mu \mathrm{H}$. The iron core should be laminated allowing pulsed operation. This provides a very elegant method to shield the septum field from the stored beam using a copper sheet between the injected and the stored beam (fig. 11). If, for instance, the half-wave pulse driving the septum has a length of $\tau=50 \mu$ s the lowest fourier-frequency will be $f_{1}=10 \mathrm{kHz}$. An electromagnetic field of that frequency has a skin-depth in copper of

$$
d=\frac{1}{\sqrt{\pi f_{i} \sigma \mu_{0}}}=0.655 \mathrm{~mm}
$$

Using a copper sheet with a thickness of at least $D=3 \mathrm{~mm}$ only $1 \%$ of the perpendicular component of the septum field will penetrate the copper. Since the perpendicular component is only a small portion of the entire field strength this would be sufficient under all running conditions. This technique is used for the pulsed septum magnets in DORIS II [6] and has demonstrated the expected results.

\section{6. rf System}

The energy loss per revolution is only $U_{0}=265.3 \mathrm{eV}$ and therefore the rf accelerating system which compensates this loss needs only a very low voltage. The cavity is therefore a simple design without any sophisticated requirements. In particular the shunt impedance can be relatively low, i.e., less than $10 \mathrm{k} \Omega$. Using an rf frequency of $f_{r f}=714 \mathrm{MHz}$, which is also used by the other damping rings [3], one can design this cavity as a loaded $\lambda / 4$ coaxial resonator (fig. 12). Such a device can easily be implemented in one of the drift sections of the predamping ring (fig. 8). 
The inner conductor has to have large transverse dimensions because of the large beam size in this region.

The maximum number of positrons captured by this ring is supposed to be $1 \times 10^{11}$ particles corresponding to an average beam current of $670 \mathrm{~mA}$. In order to get rid of instabilities the entire vacuum system has to have a low impedance and in particular the cavity should be built using a material with low conductivity, for instance stainless steel. The other important $r f$ related parameters are shown in table 4.

Table 4. rf-related Parameters

\begin{tabular}{lll}
\hline rf Frequency & $f_{r f}=714 \mathrm{MHz}$ \\
Momentum compacting factor & $\alpha=8.5 \times 10^{-3}$ \\
Harmonic number & $q=17$ \\
Energy loss/turn & $U_{0}=265.3 \mathrm{eV}$ \\
Synchrotron damping time & $\tau_{E}=8.92 \mathrm{msec}$ \\
Synchrotron lifetime & $\tau_{s y n}=50 \mathrm{hrs}$ \\
Minimum cavity voltage & $U_{m i n}=352 \mathrm{~V}$ \\
Phase angle & $\psi=49^{\circ}$ \\
Synchrotron frequency & $f_{s y n}=9.67 \mathrm{kHz}$ \\
Maximum beam current & $I$ & $=670 \mathrm{~mA}$ \\
Cavity shunt impedance & $R_{s}=10 \mathrm{k} \Omega$ (assumption) \\
Minimum rf power & $N=185 \mathrm{~W}$ \\
\hline
\end{tabular}

\section{Conclusions}

The general aim of this study was to investigate whether an emittance damping time of the order of $10 \mathrm{msec}$ is possible in the $100 \mathrm{MeV}$ range by use of a small predamping ring. This has been demonstrated by the SNAKE ring presented in this paper. The most important design criteria for such a device are also presented. The conclusion is that a sufficient emittance damping of the positron beam at an energy around $E=100 \mathrm{MeV}$ is in principal obtainable. 
There are still, however, some open questions. For instance, it has not yet been demonstrated that the betatron and energy acceptance is sufficient, because of the large energy spread of $\Delta p / p=10 \%$ particle tracking studies including higher order effects are required. Another important item is the detailed design of the SNAKEwiggler magnet itself, including field calculations. But the present design has shown no inherent problems and justifies further detailed study.

\section{Acknowledgements}

I would like to thank S. Ecklund, R. Miller, and H. Wiedemann for many fruitful discussions. I also wish to thank M. A. Allen, A. Chao and A. Hutton for reviewing the manuscript and several helpful suggestions. 


\section{References}

[1] B. Richter, The SLAC Linear Collider, Proc. XI th International Conference on High Energy Accelerators, Geneva, 1980, p. 168.

[2] SLAC Linear Collider, Conceptional Design Report, SLAC Report 229, June 1980.

[3] G. E. Fischer, W. Davies-White, T.Fieguth, H. Wiedemann, "A $1.2 \mathrm{GeV}$ Damping Ring Complex for the Stanford Linear Collider," Proc. XII ${ }^{\text {th }}$ International Conference on High Energy Accelerators, Chicago, 1983.

[4] M. Sands, "The Physics of Electron Storage Rings," International School of Physics, 1971.

[5] A. Hofmann, "Characteristics of Synchrotron Radiation from Wigglers and Undulators," New Rings Workshop, SSRL Report 83/02, October 1983.

[6] A. Febel, DESY, private communication. 


\section{Figure Captions}

1. Standard wiggler magnet.

2. Principle of the "SNAKE wiggler."

3. Magnet structure of the predamping ring using two SNAKE wigglers.

4. Cross section of the combined SNAKE wiggler sketched in fig. 3 .

5. Amplitude and dispersion functions of the damping ring optics.

6. Chromaticity compensation by use of a pole face curvature.

7. Chromaticity compensation by use of pole face rotation $(Q D)$ or pole face curvature (SNAKE wiggler).

8. Arrangement of the injection and extraction elements and of the accelerating cavity in the drift spaces of the predamping ring.

9. Vertical injection into the predamping ring.

10. Cross section of the fast vertical kicker magnet.

11. Cross section of the pulsed vertical septum magnet.

12. Coaxial cavity of the predamping ring. 


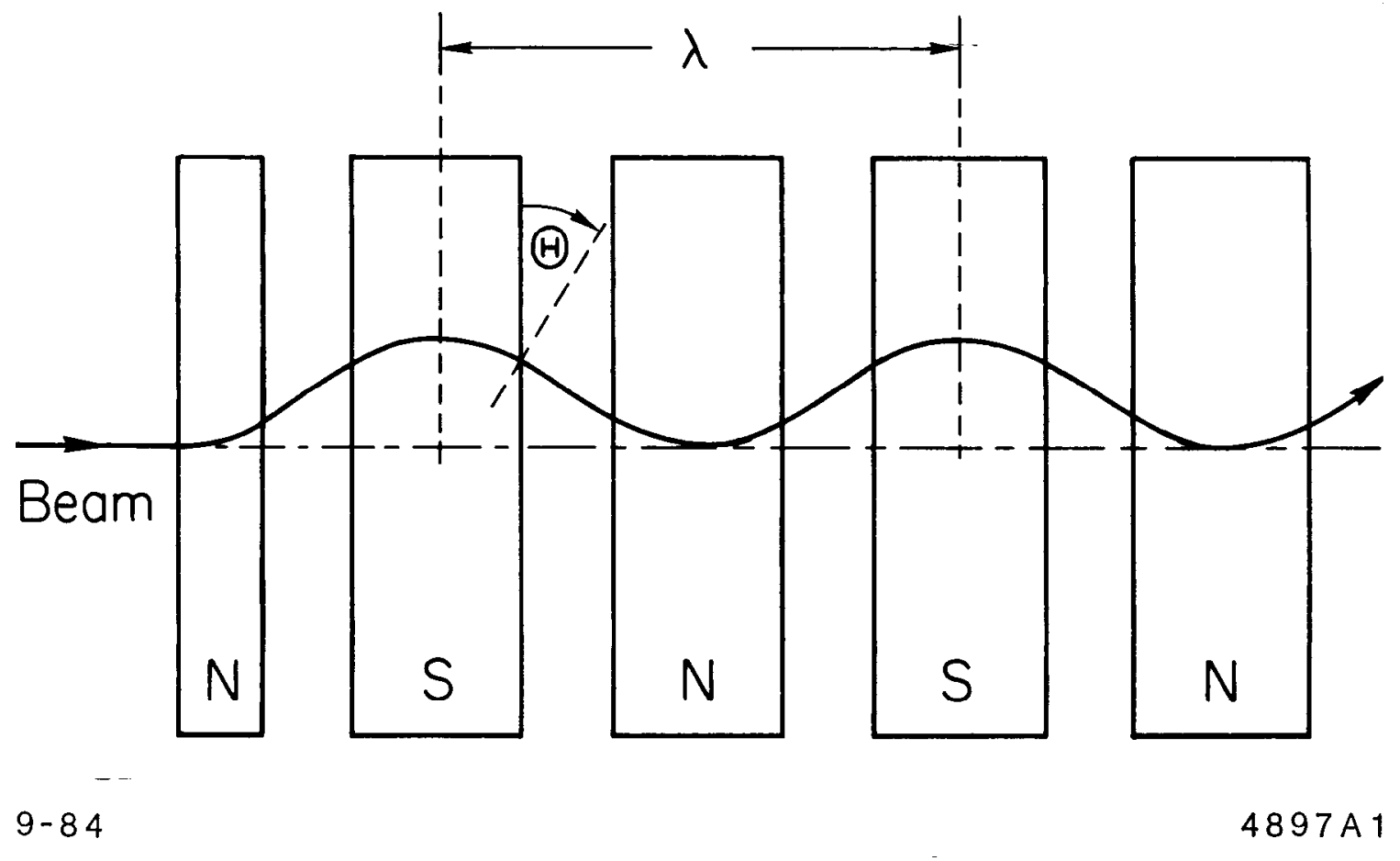

Fig. 1 


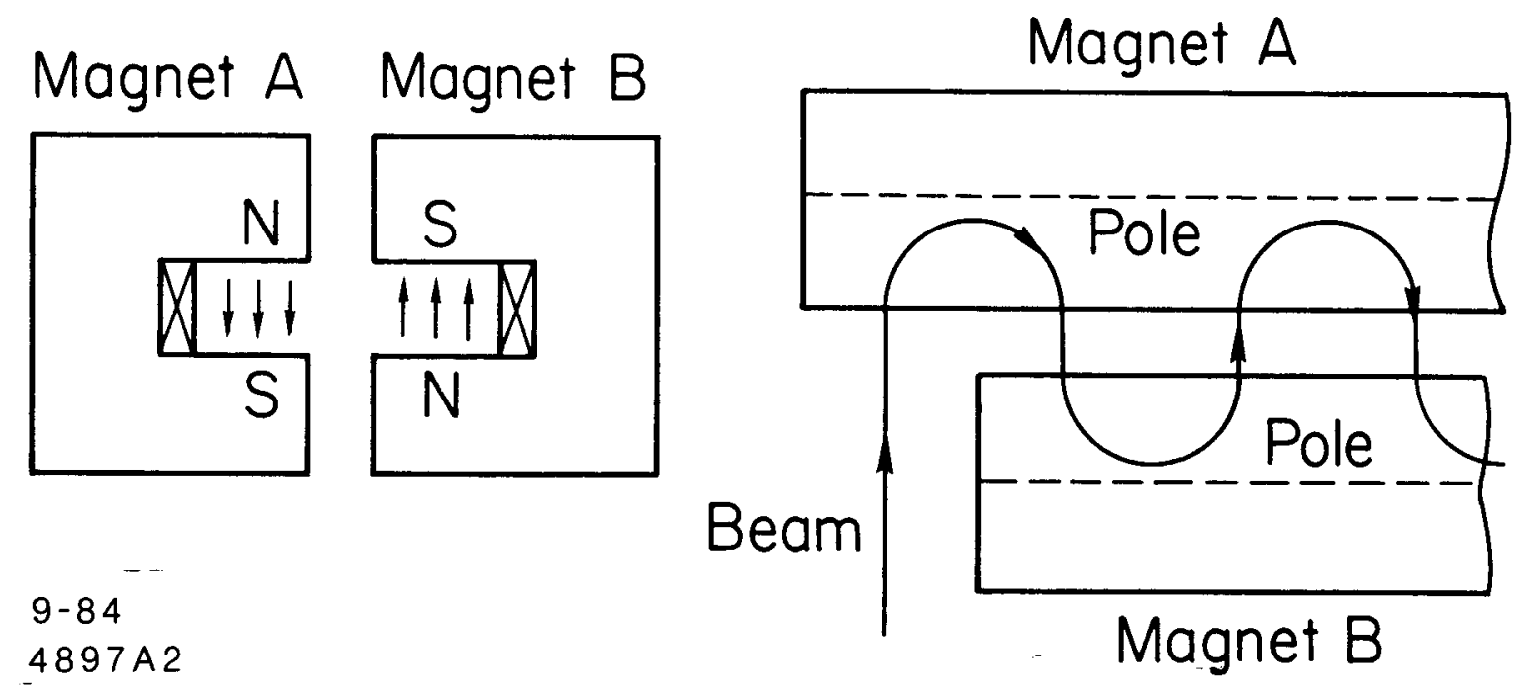

Fig. 2 


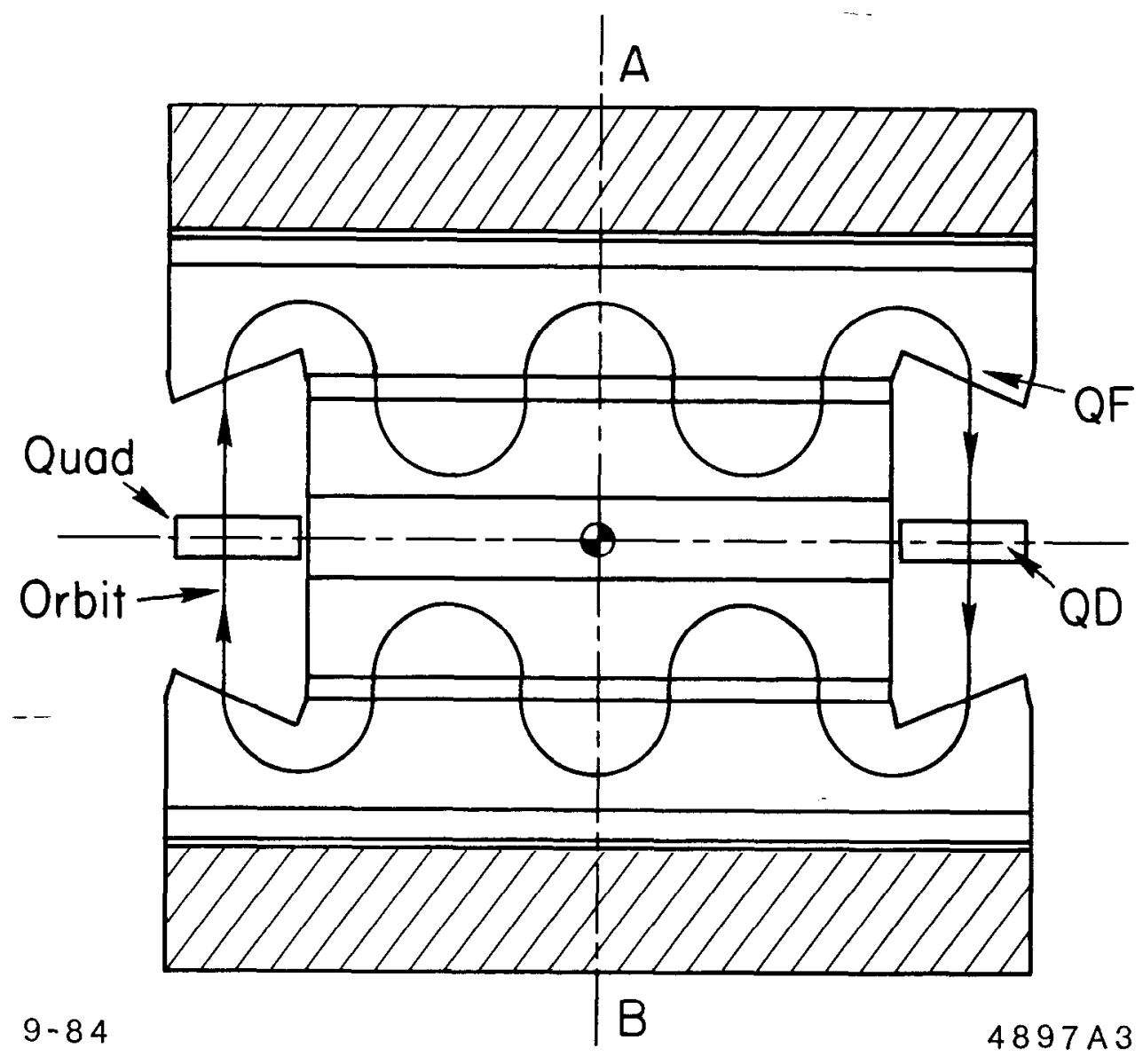

Fig. 3 


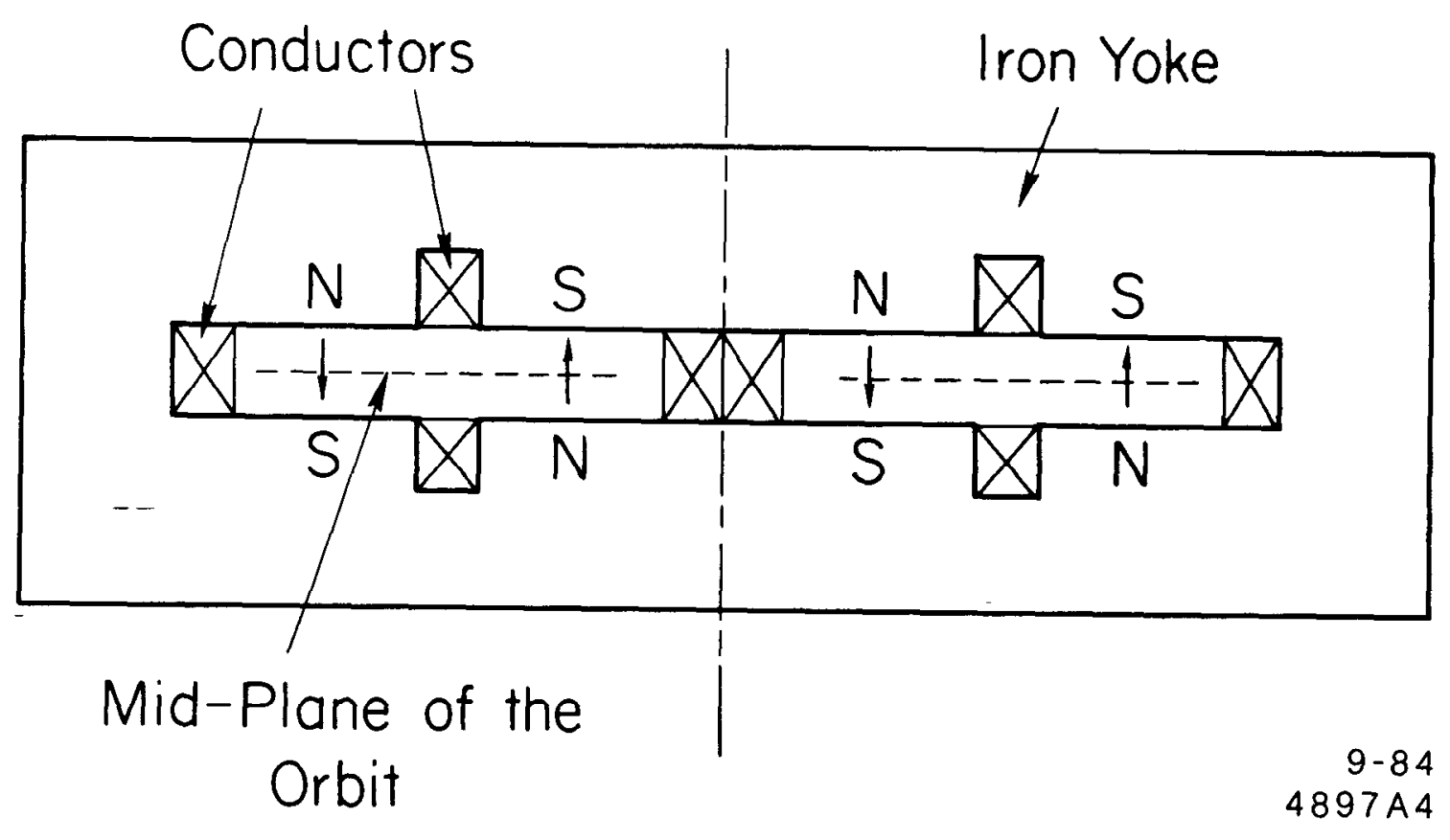

Fig. 4 


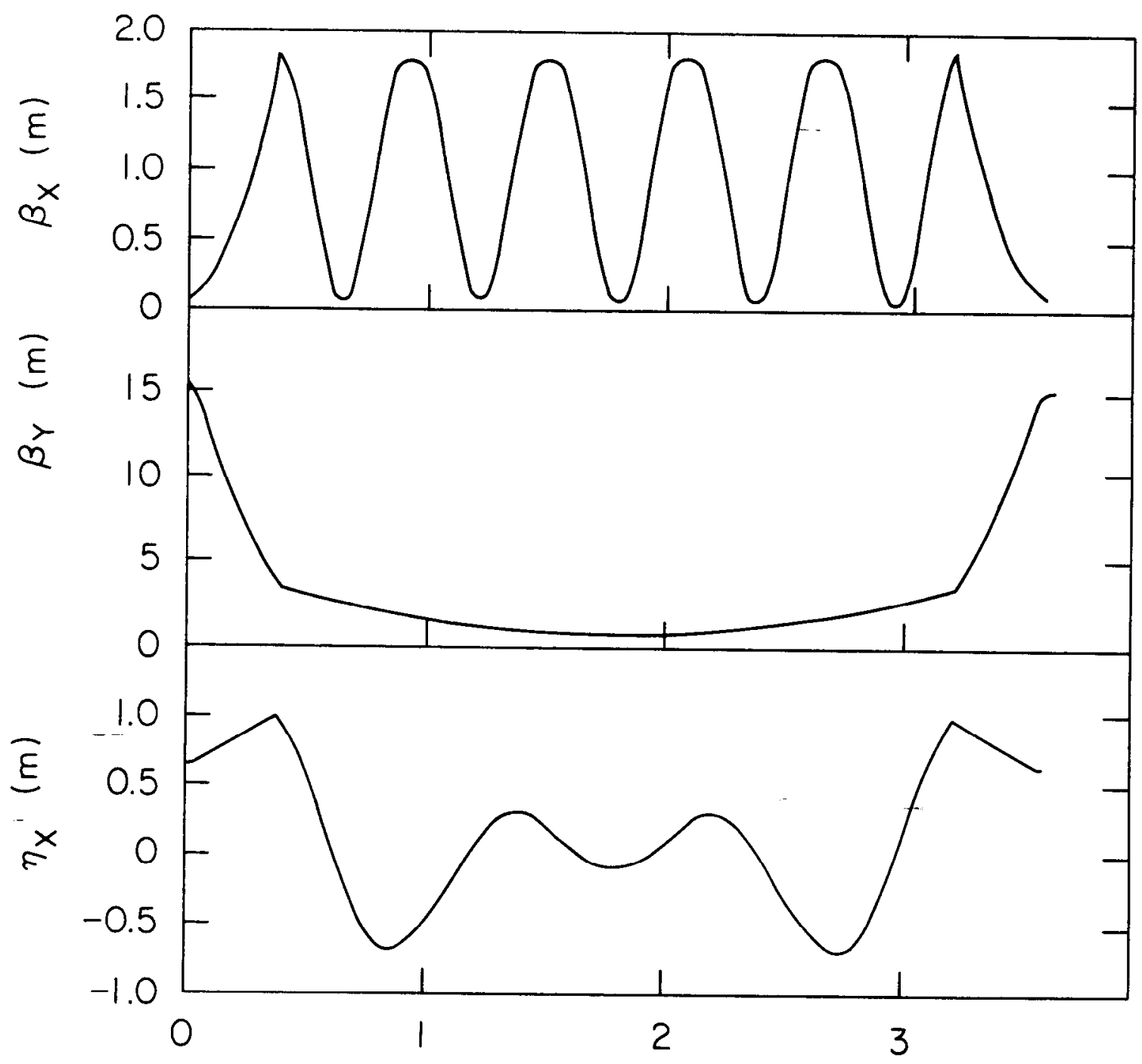

$9-84$

SNAKE DAMPING RING $(E=100 \mathrm{MeV}) \quad 4897 \mathrm{~A} 5$

Fig. 5 


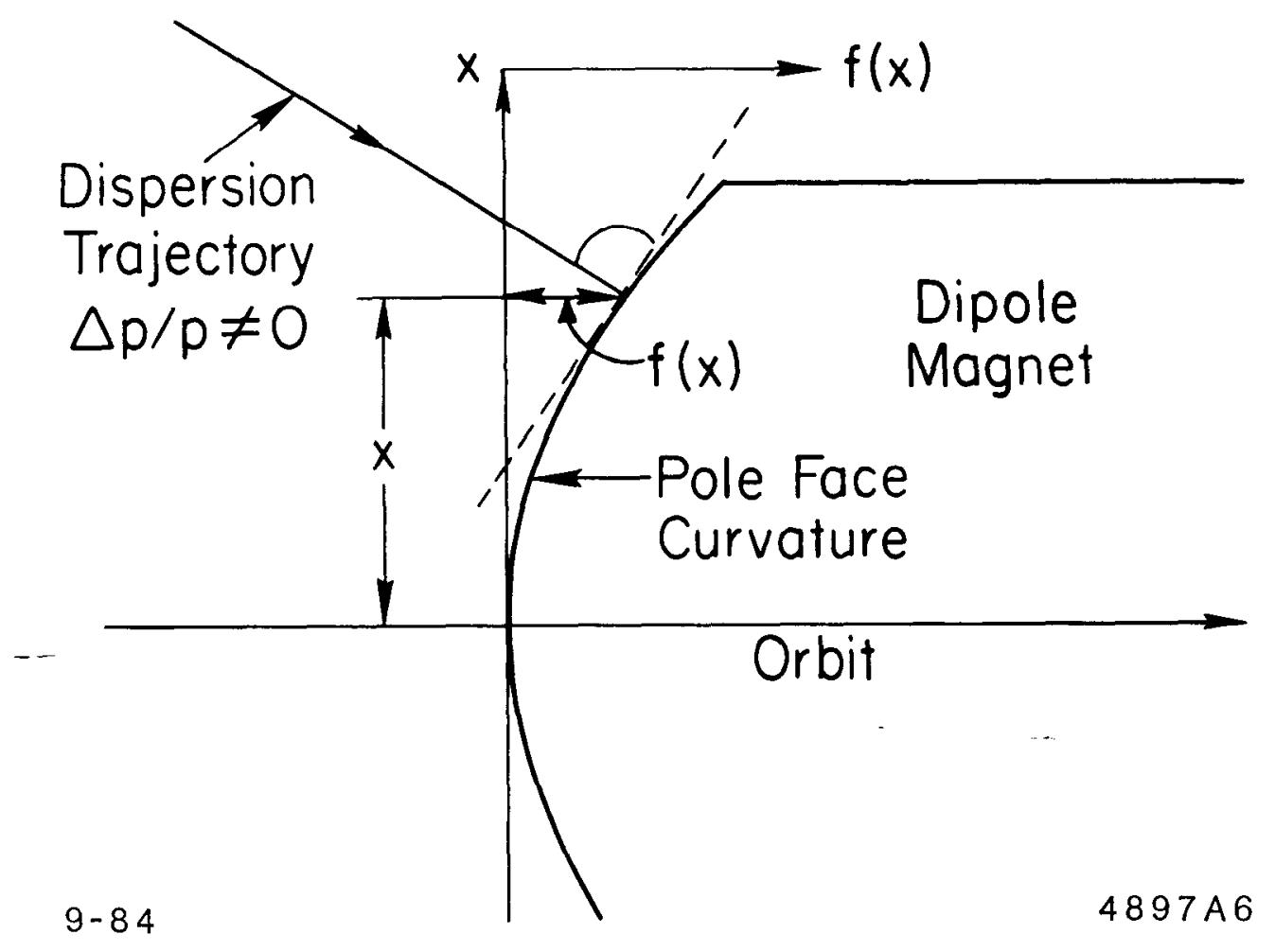

Fig. 6 


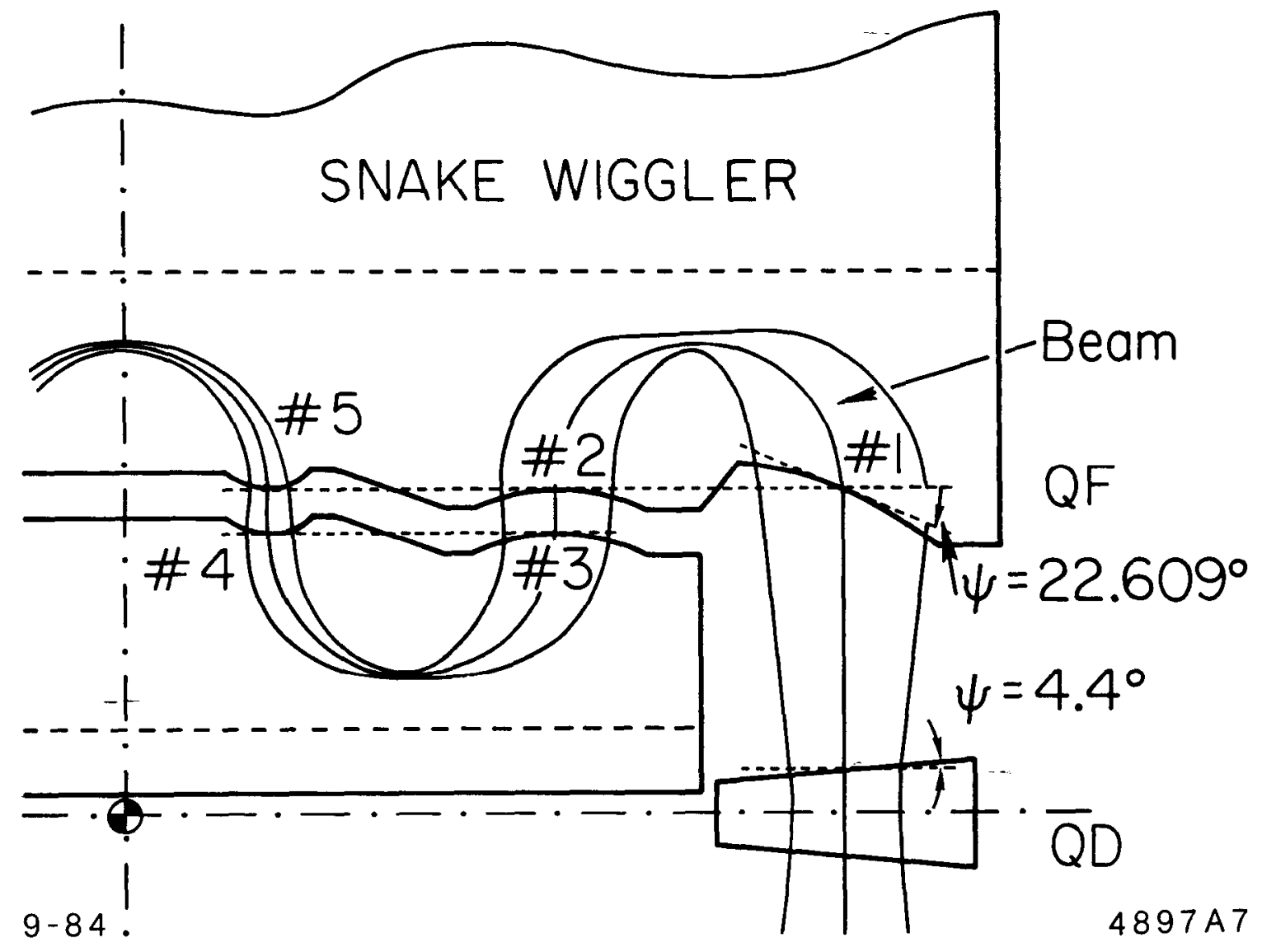

Fig. 7 


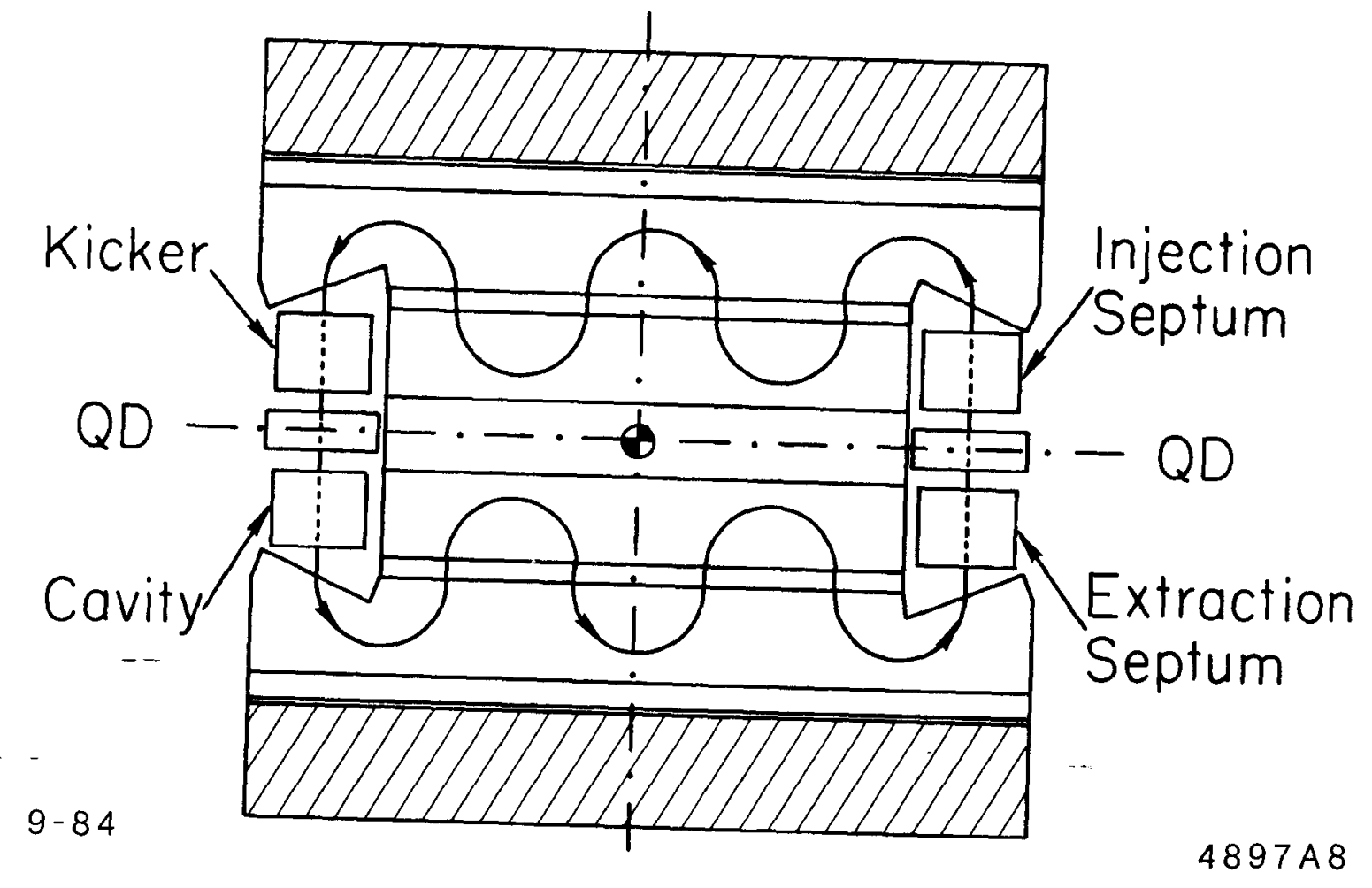

Fig. 8 


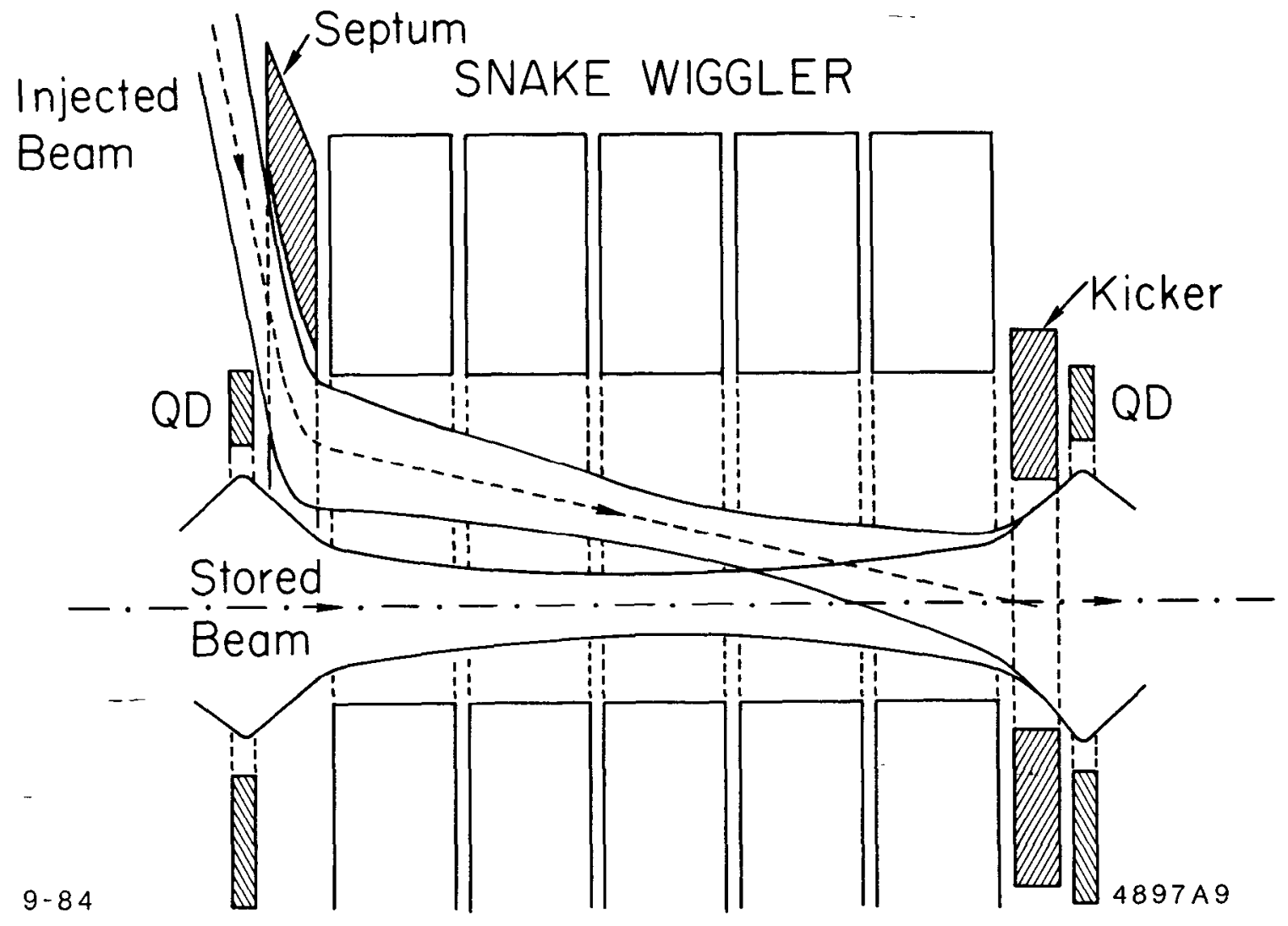

Fig. 9 


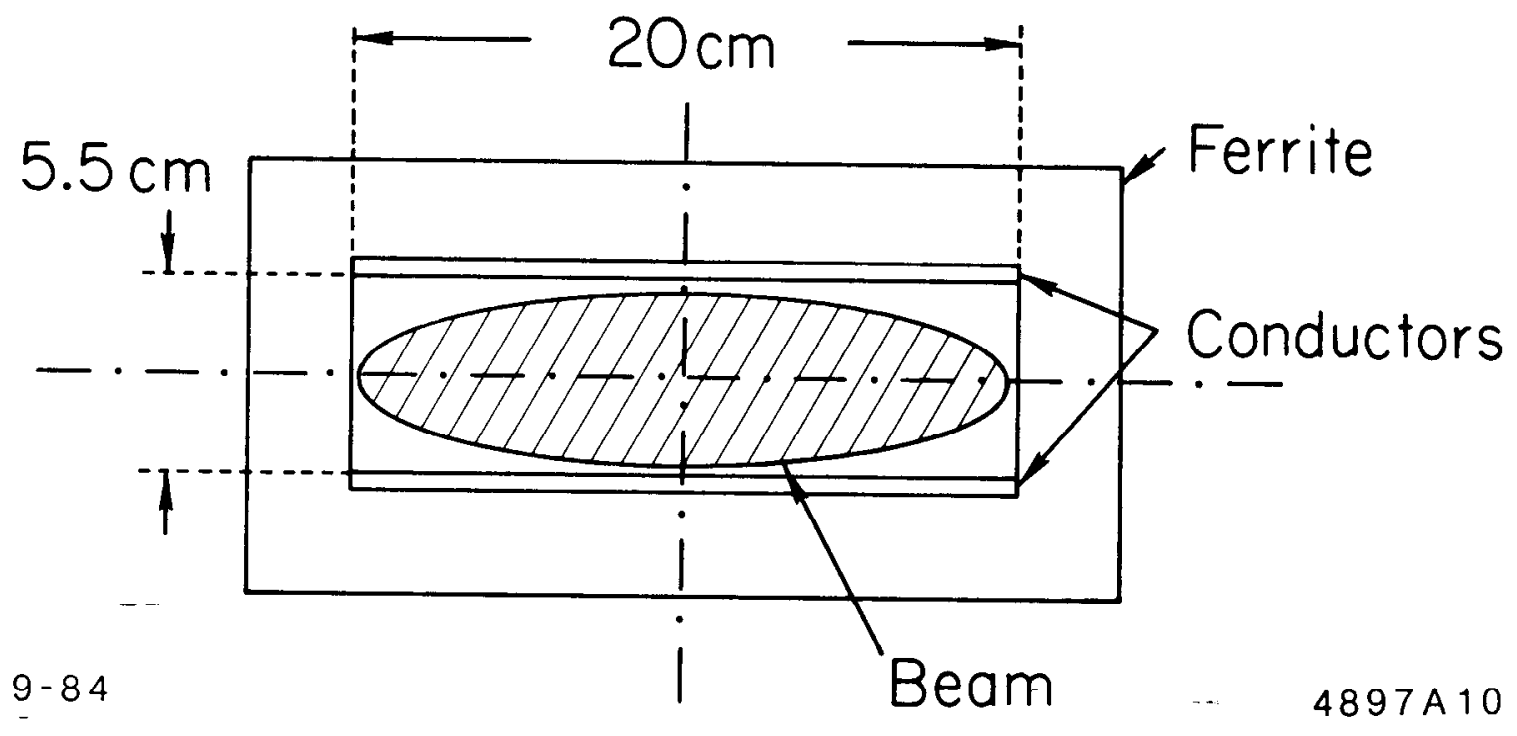

Fig. 10 


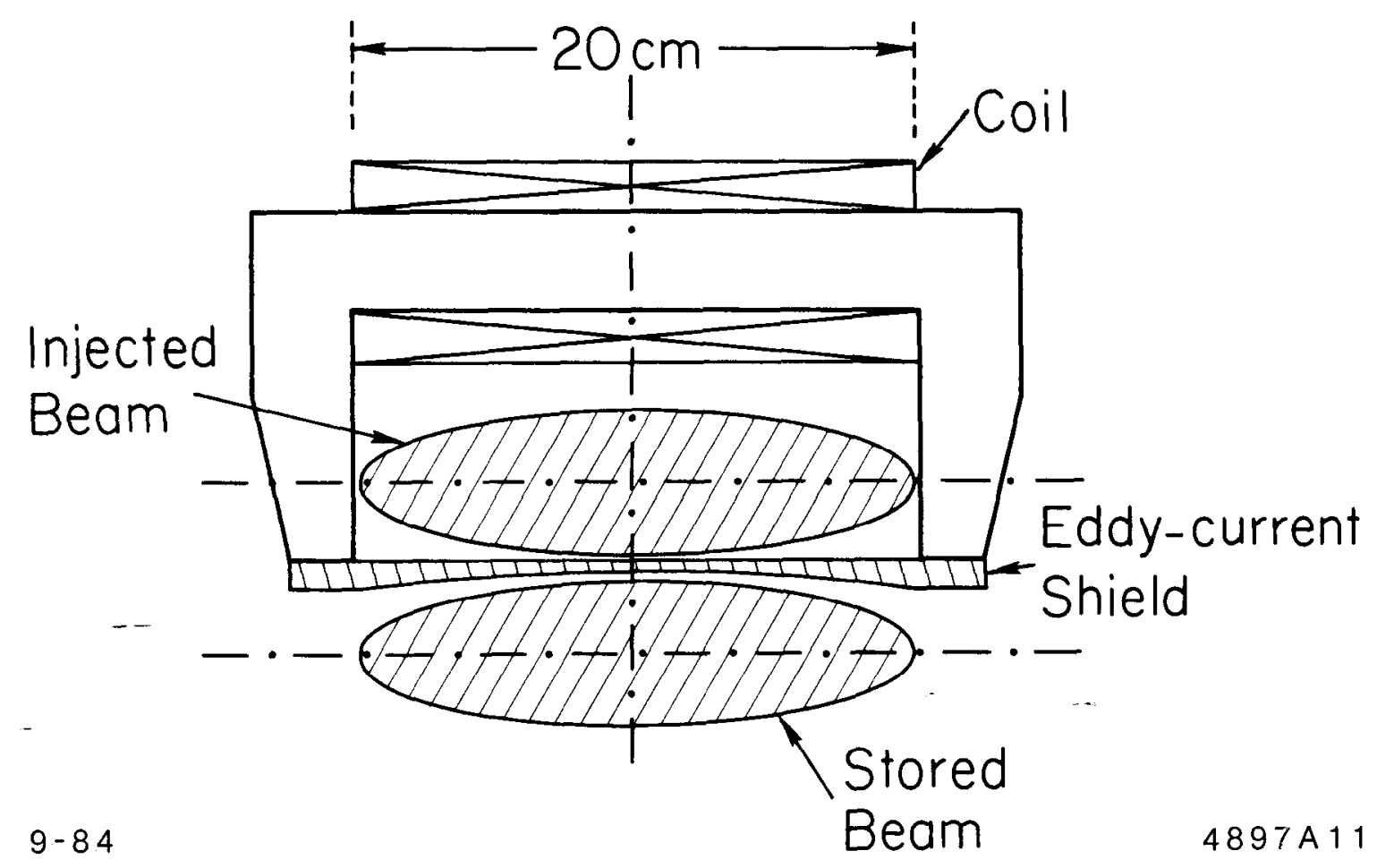

Fig. 11 


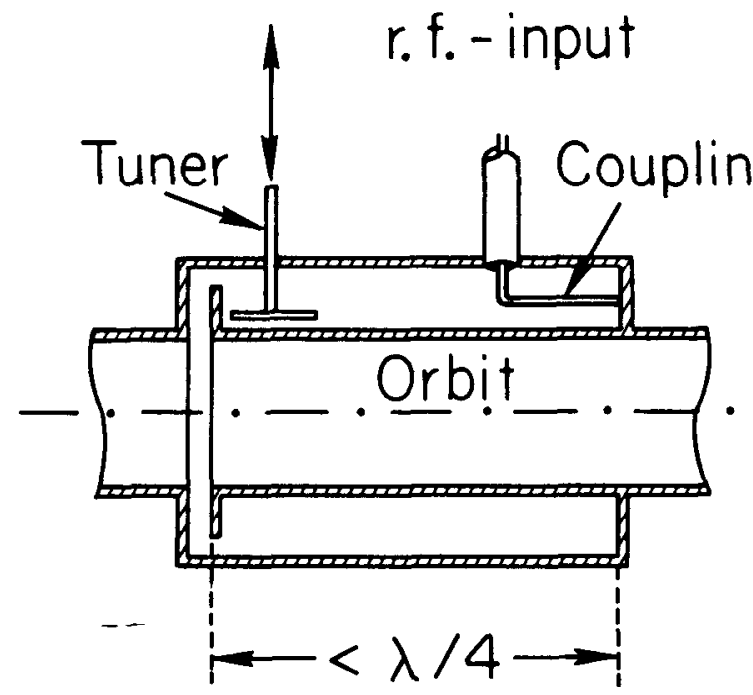

$9-84$

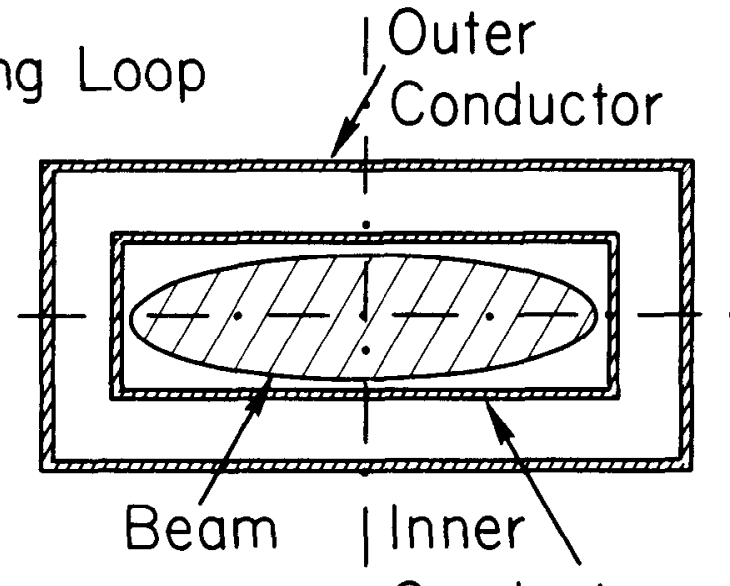

Conductor

4897 A 12

Fig. 12 